This item was submitted to Loughborough's Research Repository by the author.

Items in Figshare are protected by copyright, with all rights reserved, unless otherwise indicated.

\title{
Effective image clustering based on human mental search
}

PLEASE CITE THE PUBLISHED VERSION

https://doi.org/10.1016/j.asoc.2019.02.009

PUBLISHER

(c) Elsevier

VERSION

AM (Accepted Manuscript)

PUBLISHER STATEMENT

This paper was accepted for publication in the journal Applied Soft Computing Journal and the definitive published version is available at https://doi.org/10.1016/j.asoc.2019.02.009

LICENCE

CC BY-NC-ND 4.0

\section{REPOSITORY RECORD}

Mousavirad, Seyed Jalaleddin, Hossein Ebrahimpour-Komleh, and Gerald Schaefer. 2019. "Effective Image Clustering Based on Human Mental Search". figshare. https://hdl.handle.net/2134/37402. 


\title{
Effective Image Clustering based on Human Mental Search
}

\author{
Seyed Jalaleddin Mousavirad ${ }^{1}$, Hossein Ebrahimpour-Komleh ${ }^{1}$ and Gerald Schaefer ${ }^{2}$ \\ ${ }^{1}$ Department of Electrical and Computer Engineering, University of Kashan, Iran \\ ${ }^{2}$ Department of Computer Science, Loughborough University, U.K.
}

\begin{abstract}
Image segmentation is one of the fundamental techniques in image analysis. One group of segmentation techniques is based on clustering principles, where association of image pixels is based on a similarity criterion. Conventional clustering algorithms, such as $k$-means, can be used for this purpose but have several drawbacks including dependence on initialisation conditions and a higher likelihood of converging to local rather than global optima.

In this paper, we propose a clustering-based image segmentation method that is based on the human mental search (HMS) algorithm. HMS is a recent metaheuristic algorithm based on the manner of searching in the space of online auctions. In HMS, each candidate solution is called a bid, and the algorithm comprises three major stages: mental search, which explores the vicinity of a solution using Levy flight to find better solutions; grouping which places a set of candidate solutions into a group using a clustering algorithm; and moving bids toward promising solution areas. In our image clustering application, bids encode the cluster centres and we evaluate three different objective functions.

In an extensive set of experiments, we compare the efficacy of our proposed approach with several state-of-the-art metaheuristic algorithms including a genetic algorithm, differential evolution, particle swarm optimisation, artificial bee colony algorithm, and harmony search. We assess the techniques based on a variety of metrics including the objective functions, a cluster validty index, as well as unsupervised and supervised image segmentation criteria. Moreover, we perform some tests in higher dimensions, and conduct a statistical analysis to compare our proposed method to its competitors. The obtained results clearly show that the proposed algorithm represents a highly effective approach to image clustering that outperforms other state-of-the-art techniques.
\end{abstract}

Keywords: Image clustering, metaheuristic algorithms, human mental search, image segmentation.

\section{Introduction}

Image segmentation is a fundamental task in image processing and analysis. Image segmentation is the process of dividing an image into several homogeneous meaningful areas. Pixels associated with a certain area, commonly described based on features such as colour or texture, exhibit more commonalities than those assigned to different areas. Image segmentation is employed in a variety of applications, such as satellite image analysis [1, 2], colour quantisation [3], tumour detection [4], food quality evaluation [5], and modelling of microstructures [6], to name a few.

Clustering approaches [7] are among the most commonly employed methods for image segmentation. Here, an image 
is divided into a set of groups (clusters) so that the members of a group share more commonalities than those in different groups. This is typically achieved by optimising a criterion formulated as maximising the similarity among the members of a cluster, while ensuring that clusters are distinct from each other.

The $k$-means algorithm is the most commonly employed clustering algorithm. Here, first $k$ cluster centres are defined randomly. Then, using a similarity criterion such as Euclidean distance, every pattern is assigned to its closest cluster centre. After that, the location of every cluster centre is recalculated. The process continues until a stopping condition is satisfied. $K$-means is relatively simple but is also dependent on initialisation and converges to the closest local optimum [8].

Clustering can also be formulated as an optimisation problem. An optimisation problem is the problem of finding the minimum (or maximum) of an objective function in search space. Since many optimisation techniques have elements (such as initialisation) that introduce randomness, population-based metaheuristic algorithms, which use of multiple solutions and their collaboration, can be employed for improved performance [8]. Metaheuristic algorithms are also problem-independent and can thus be used for a broad spectrum of optimisation problems. Using a series of operators, such algorithms try to direct a set of initially random solutions toward an appropriate area of the search space. Examples of population-based metaheuristic algorithms include genetic algorithms [9], particle swarm optimisation [10], differential evolution [11], bee colony algorithms [12], and harmony search [13].

In solving an optimisation problem using a metaheuristic algorithm, three significant issues should be taken into account: (1) the structure of a solution; (2) the objective function which determines the quality of a solution; and (3) the operators which cause the initial random solutions in search space to be directed to form better solutions. The operators have a crucial effect on the efficacy of a metaheuristic algorithm, so that the difference between metaheuristic algorithms is expressed by their different operators. Many studies have also been conducted to improve the effectiveness of different operators using new metaheuristic algorithms or extensions of existing algorithms for image segmentation [14-16].

One of the challenges in image clustering, compared with other applications, is that there is a large number of samples. For example, in a relatively small image of size $256 \times 256$, the number of samples (pixels) is 65,536 , while an image of size $512 \times 512$ comprises 262,144 pixels. In the literature, various work has proposed the use of population-based metaheuristic algorithms for image clustering. For example, [17] used fuzzy c-means (FCM) and genetic algorithms (GAs) for image clustering with an objective function based on the total weighted squared error. This method showed a higher efficacy on satellite images compared with four algorithms including a self-organising map (SOM), a hybrid genetic algorithm (HGA), FCM, and a combination of SOM and HGA. [18] studied colour image segmentation using a GA and a SOM where the latter was used as a pre-processing operation to identify the number of clusters and an objective function based on compactness and separation. The obtained results indicated performance exceeding those of other state-of-the-art algorithms.

Particle swarm optimisation (PSO) [10, 19] is a population-based algorithm inspired by the social behaviour of birds. The first PSO-based image clustering algorithm is reported in [20]. Here, the structure of each particle is a singledimensional array where every component corresponds to a cluster centre, and an error function was used as objective function. Tested on four images, in comparison with the ISODATA algorithm, the PSO approach showed a higher efficacy. 21] proposed a chaos-based PSO algorithm for texture image segmentation where the application of chaos prin- 
ciples aims at avoiding local optima. The obtained results indicated that this method was able to outperform standard PSO. In [22], a bare-bones PSO (BPSO) algorithm was studied for image clustering. This approach does not require parameter tuning, and good performance in comparison to other algorithms such as $k$-means and PSO was observed. Other PSO-based image clustering algorithms include [23-26], while other population-based metaheuristic algorithms such as differential evolution [27,-29], bee colony algorithms [14,30] and harmony search [31] have also been proposed for image clustering. Although these algorithms have shown satisfactory results in comparison with conventional algorithms, still better performance is required. Despite the diverse work done in this area, this is still a very active research subject with the aim of improving the efficacy of image clustering algorithms.

The human mental search (HMS) algorithm [32] is a relatively recent population-based algorithm which has demonstrated competitive performance for solving optimisation problems. Every solution in HMS is called a bid. The algorithm has three main operators. The first operator is mental search, which searches the vicinity of a solution using Levy flight. Levy flight, a type of random walk with varying step size, can simultaneously improve diversification and intensification. The second HMS operator is grouping which clusters the population with the aim of identifying promising areas in search space. Finally, the third operator instigates movement of solutions towards these promising areas.

According to the No Free Lunch (NFL) theorem [33] there is no best algorithm for solving an optimisation problem. This theorem is one of the main motivations for applying various metaheuristic algorithms for problems such as clustering [27, 30, 31]. The HMS algorithm as a novel metaheuristic yielded satisfactory performance in comparison with other algorithms such as PSO, harmony search, and artificial bee colony algorithm [32].

Based on the NFL theorem and the competitive performance of HMS, in this paper, we propose a novel effective method for image clustering using the HMS algorithm. We define the structure of each HMS bid to be an array whose length equals the number of image clusters. For the optimisation, we investigate three objective functions, where the first two are based on intra-cluster distance, inter-cluster separation and quantisation error, while the third also incorporates a mean squared error criterion.

In an extensive set of experiments, we compare the efficacy of our proposed approach with several state-of-the-art metaheuristic algorithms including a genetic algorithm, differential evolution, particle swarm optimisation, artificial bee colony algorithm, and harmony search, and demonstrate it to exhibit excellent image clustering performance.

The remainder of the paper is organised as follows. Section 2 describes the concept of clustering, while Section 3 introduces the human mental search algorithm. Our proposed algorithm is described in Section 4, while experimental results are presented in Section 5. Finally, Section 6 concludes the paper.

\section{Pattern clustering}

The purpose of clustering is to group a set of patterns so that the members of each group have more similarity compared to intergroup members. Mathematically, clustering is defined as follows. Suppose, we have a set $P$ comprising $N d$ dimensional patterns, $P=\left\{p_{1}, p_{2}, \ldots, p_{n}\right\}$. In the context of this paper, a pattern is equivalent to a pixel value. The aim of clustering is to divide $P$ into $K$ clusters $C=\left\{c_{1}, c_{2}, \ldots, c_{K}\right\}$ so that the following conditions are satisfied:

1. Each cluster contains at least one pattern $c_{i} \neq \emptyset, i=1 \ldots K$; 
2. The total number of patterns in all clusters is equal to the total number of patterns in the dataset: $\bigcup_{i=1}^{k} c_{i}=\mathrm{P}$;

3. Different clusters should not have mutual patterns: $c_{i} \cap c_{j}=\varphi, i, j=1 \ldots K, i \neq j$.

A criterion needs to be defined to be able to assess the clustering quality. The mean squared error (MSE) is the most common function for this purpose and defined as

$$
f(P, C)=\sum_{j=1}^{K} \sum_{P_{i} \in C_{j}} d\left(p_{i}, c_{j}\right),
$$

where $d\left(p_{i}, c_{j}\right)$ is the distance between pattern $p_{i}$ pattern and its cluster center $c_{j}$. Several distance criteria can be used to calculate the dissimilarity between patterns with the Euclidean distance

$$
d\left(p_{i}, p_{j}\right)=\sqrt{\sum_{m=1}^{d}\left(p_{i}^{m}-p_{j}^{m}\right)^{2}},
$$

where $p_{i}^{m}$ is the $m$-th dimension of $p_{i}$, being the most popular one.

The $k$-means algorithm is the best known and most employed algorithm in data clustering. It proceeds in the following steps:

Step 1: Randomly select $K$ points as cluster centres;

Step 2: Allocate each pattern to its closest cluster centre. More formally, if $C$ is the set of cluster centres, then each pattern $x$ is allocated to a cluster centre based on:

$$
\underset{c_{i} \in C}{\arg \min } d\left(x, c_{i}\right)
$$

where $d$ is the Euclidean distance;

Step 3: Recalculate the position of each cluster centre as the centroid of its allocated patterns as

$$
c_{i}=\frac{1}{\left|S_{i}\right|} \sum_{x \in S_{i}} x
$$

where $S_{i}$ is the $i$-th cluster;

Step 4: Repeat Steps 2 and 3 until convergence (or until a different stopping criterion is met).

\section{Human mental search}

Human mental search [32] is a recently introduced population-based metaheuristic algorithm for solving optimisation problems. Every solution in HMS is called a bid and the algorithm is inspired by the manner of searching in a bid space of online auctions.

Algorithm 1 summarises the HMS algorithm in form of pseudo-code while in the following we describe the algorithm in more detail. 


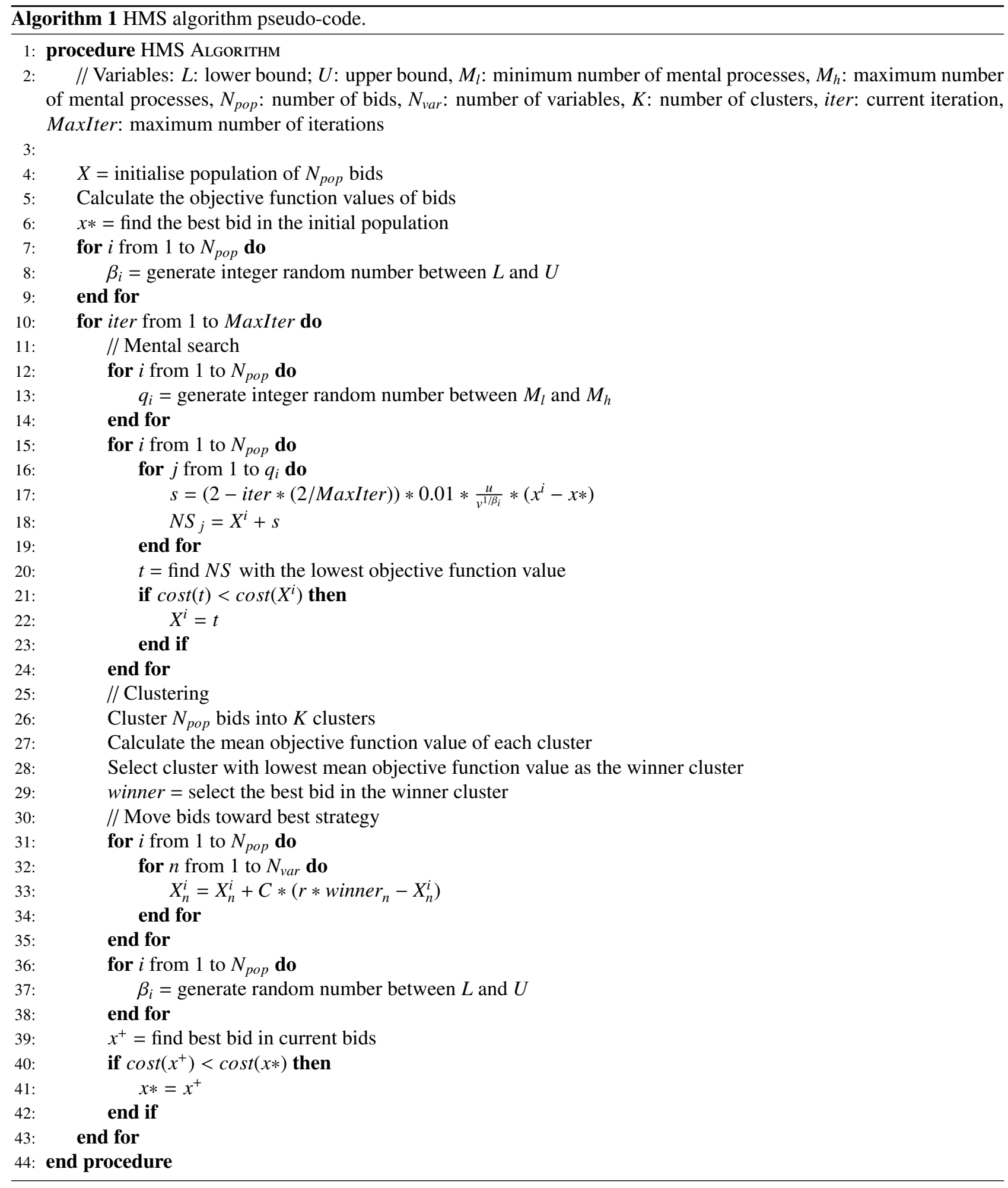

\subsection{Creating primary bids}

Like other population-based algorithms, HMS commences by creating a set of $N_{\text {pop }}$ random candidate solutions. Every solution is called a bid. In a $d$-dimensional space, a bid is defined as

$$
\text { bid }=\left[x_{1}, x_{2}, \ldots, x_{d}\right] .
$$


A bid is assessed using an objective function $f()$ that evaluates the quality of a candidate solution:

$$
f(\text { bid })=f\left(x_{1}, x_{2}, \ldots, x_{d}\right)
$$

\subsection{Mental search}

Mental search is one of the main operators introduced in the HMS algorithm. This operator creates a series of new solutions around each bid using a random walk in which the step size follows a Levy distribution. Compared to Brownian motion, use of the Levy distribution allows for improved diversification (due to 'long jumps') and intensification (due to 'small steps') and is thus more effective in exploring unknown spaces.

A new position is obtained as

$$
N S=b i d+S
$$

with $S$ is calculated as

$$
S=(2-\text { iter } *(2 / \text { MaxIter })) * \alpha \oplus \text { Levy, }
$$

where MaxIter is the maximum number of iterations, iter indicates the current iteration, and $\alpha$ is a random number, while $\oplus$ signifies element-wise multiplication. $(2-$ iter $*(2 /$ MaxIter $))$ is a descending factor, starting at 2 and ending towards 0 , that considers a larger vicinity of a solution at the beginning and smaller vicinities at the end of the algorithm, thus yielding high diversification at the beginning and high intensification at the end.

The step size $S$ using a Levy distribution is calculated as

$$
S=(2-\text { iter } *(2 / \text { MaxIter })) * \alpha \oplus \text { Levy }=(2-\text { iter } *(2 / \text { MaxIter })) * 0.01 * \frac{u}{v^{1 / \beta}} *\left(x^{i}-x *\right),
$$

where $x *$ is the best position found so far, and $u$ and $v$ are two random numbers with normal distributions as

$$
u \sim N\left(0, \sigma_{u}^{2}\right), \quad v \sim N\left(0, \sigma_{v}^{2}\right)
$$

and

$$
\sigma_{u}=\left\{\frac{\Gamma(1+\beta) \sin \left(\frac{\pi \beta}{2}\right)}{\Gamma\left[\left(\frac{1+\beta}{2}\right)\right] \beta 2^{(\beta-1) / 2}}\right\}^{1 / \beta}, \sigma_{v}=1,
$$

where $\Gamma$ is a standard gamma function.

Figure 1 illustrates the mental search operator for two out of 12 existing bids (black dots) in the search space. The numbers of new bids created for bid A and B are 4 and 5 respectively (selected randomly). As can be seen, the vicinity of a solution is searched with the aim of finding better solutions (red and blue dots) and there are some 'large jumps'. Finally, each bid is superseded with the best bid created by the mental search operator. 


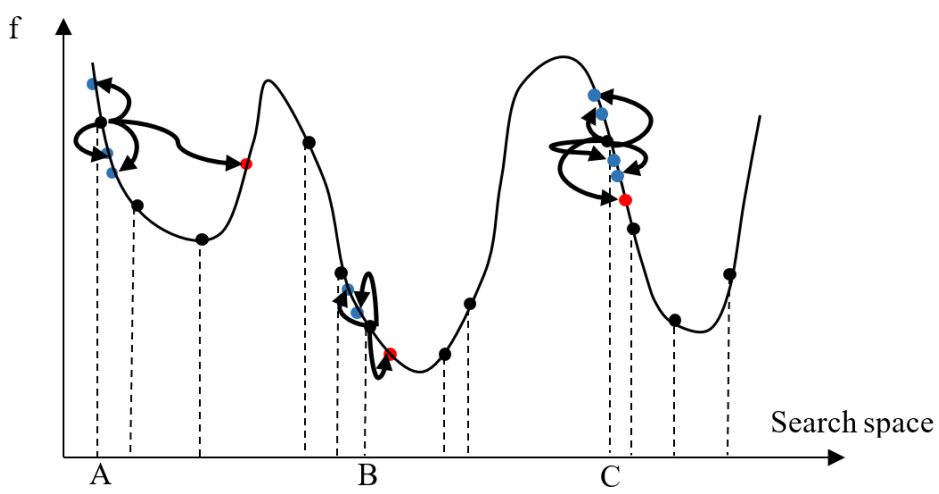

Figure 1: Example of mental search operator.

\subsection{Grouping bids}

The second operator in the HMS algorithm is a solution grouping operator using a clustering method. Bid clustering would cause solutions that are close to each other to be placed in one cluster. Here, the $k$-means clustering operator is used for bid clustering. After clustering, the mean objective function value is calculated for each cluster to determine promising areas in search space; an area with a lower mean objective value is likely a more appropriate area. Figure 2 illustrates the grouping operator. There are 12 bids in the figure, which are divided into three clusters. As can be seen, there are closer solutions in each cluster. This mechanism is different from most algorithms, which typically use the best solutions to identify promising areas in search space.

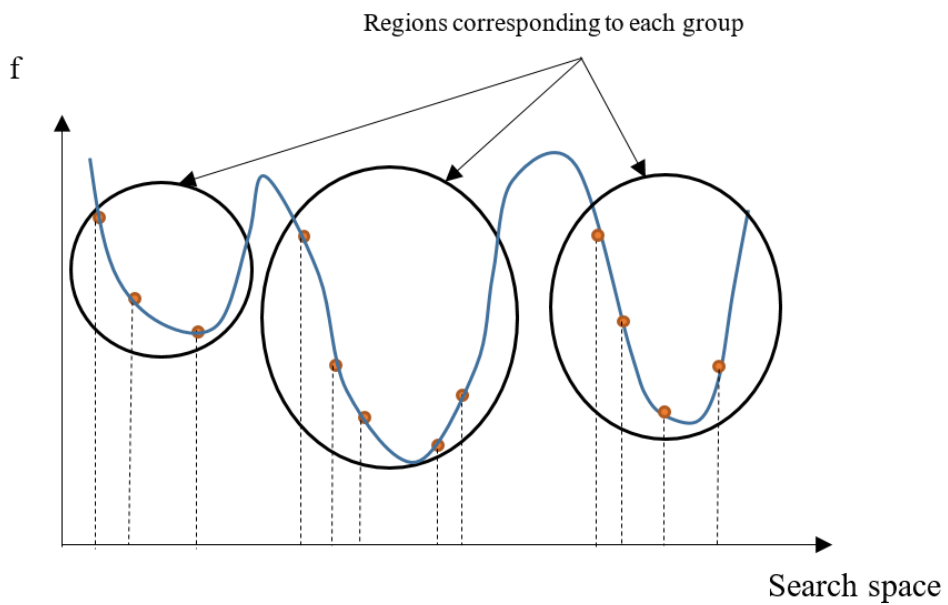

Figure 2: Example of bid grouping operator.

\subsection{Moving towards the promising area}

After grouping, the area with the lowest mean objective function value is selected as the winner group or the promising area. Then, the bids in the other groups move towards the best bid in this area. It is worth mentioning that the best bids among all bids may not belong to the winner group. Bids are updated as

$$
{ }^{t+1} \operatorname{bid}_{n}={ }^{t} \operatorname{bid}_{n}+C *\left(r \times{ }^{t} \text { winner }_{n}-{ }^{t} \text { bid }_{n}\right)
$$


where ${ }^{t+1}$ bid $_{n}$ is the $n$-th bid element at iteration $t+1,{ }^{t}$ winner $_{n}$ is the $n$-th element of the best bid in the winner group, $t$ is the current iteration, $C$ is a constant number, and $r$ is in $[0 ; 1]$ derived from a normal distribution.

\section{Image clustering using human mental search}

In this paper, we propose an image clustering algorithm based on human mental search. For this, two issues to be determined are the structure of each bid and the objective function which we define in the following, before describing our proposed method step-by-step and working through an illustrative example.

\subsection{Bid structure}

In our approach, the structure of each bid is an array which encodes the centres of the clusters. The length of the array is equal to $K$ with $K$ the number of clusters. The upper and lower bound of each bid are calculated as $L=\min (I)$ and $U=\max (I)$ where $I$ is the image. In other words, the upper and lower bound are the minimum and maximum pixel values.

For illustration, Figure 3 displays a sample image of size $10 \times 10$.

\begin{tabular}{|c|c|c|c|c|c|c|c|c|c|}
\hline 12 & 48 & 53 & 89 & 10 & 14 & 32 & 26 & 86 & 88 \\
\hline 16 & 14 & 77 & 93 & 12 & 20 & 36 & 43 & 89 & 93 \\
\hline 19 & 19 & 82 & 99 & 11 & 25 & 40 & 38 & 92 & 94 \\
\hline 25 & 120 & 94 & 48 & 30 & 18 & 29 & 49 & 94 & 95 \\
\hline 30 & 114 & 105 & 89 & 19 & 30 & 207 & 199 & 191 & 101 \\
\hline 43 & 119 & 110 & 93 & 19 & 28 & 202 & 211 & 186 & 192 \\
\hline 38 & 142 & 98 & 104 & 14 & 13 & 219 & 198 & 232 & 188 \\
\hline 126 & 163 & 96 & 91 & 98 & 110 & 200 & 190 & 225 & 185 \\
\hline 94 & 122 & 94 & 99 & 120 & 108 & 217 & 185 & 227 & 192 \\
\hline 160 & 192 & 210 & 208 & 209 & 212 & 218 & 189 & 230 & 185 \\
\hline
\end{tabular}

Figure 3: Sample image.

Here, $L=\min (I)=10$ and $U=\max (I)=232$. Suppose that the number of clusters is 3 . Hence, the length of each bid is 3 and an example of a bid would be $(193,92,126)$.

\subsection{Objective functions}

Choosing an appropriate objective function is crucial for any population-based metaheuristic algorithm, since such algorithms update candidate solutions based on their quality as established by the objective function. In our work, we investigate three objective functions:

1. The first objective function [25] combines three factors expressing error, intra-cluster distance, and inter-cluster distance in a weighted form as

$$
C F 1(\text { bid }, I)=w_{1} d_{\max }(Z, b i d)+w_{2}\left(z_{\max }-d_{\min }(I, b i d)\right)+w_{3} J_{e},
$$


where $I$ is the image, bid is a bid defined as bid $=\left[m_{1}, m_{2}, \ldots, m_{K}\right]$ with $m_{i}$ indicating the $i$-th cluster centre, $z_{\max }$ is the maximum data value (for an image of bitdepth $s, z_{\max }=2^{s}-1$ ), and $w_{1}, w_{2}$, and $w_{3}$ are user-defined constants. $d_{\max }(Z, b i d)$, the intra-cluster distance, is calculated as

$$
d_{\max }(Z, b i d)=\max _{j=1, \ldots, K} \sum_{\forall p_{i} \in c_{j}} \frac{d\left(p_{i}, b i d\right)}{\left|c_{j}\right|}
$$

where $K$ is the number of clusters, $\left|c_{j}\right|$ is the number of members of the $j$-th cluster, and $d\left(p_{i}, b i d\right)$ is the Euclidean distance between $p_{i}$ and bid.

The inter-cluster separation $d_{\min }($ bid $)$, which is based on the minimum average Euclidean distance between a pair of clusters and should be maximised, is defined as

$$
d_{\min }(\text { bid })=\min _{\forall i, j, i \neq j}\left(d\left(m_{i}, m_{j}\right)\right)
$$

The quantisation error $J_{e}$ is calculated as

$$
J_{e}=\frac{\sum_{j=1}^{K} \sum_{\forall p_{i} \in c_{j}} d\left(p_{i}, \text { bid }\right) /\left|c_{j}\right|}{K} .
$$

Following [14], we set $w_{1}, w_{2}$, and $w_{3}$ to $0.4,0.2$, and 0.4 , respectively.

2. To avoid having to find appropriate values for $w_{1}, w_{2}$, and $w_{3}$, the second objective function [22] is defined as

$$
C F 2(\text { bid }, I)=\frac{d_{\max }(I, b i d)+J_{e}}{d_{\min }(I, b i d)} .
$$

3. The third objective function [14] is defined as

$$
C F 3(b i d, I)=J_{e} \times \frac{d_{\max }(I, b i d)}{d_{\min }(I, b i d)} \times\left(d_{\max }(I, b i d)+z_{\max }-d_{\min }(I, b i d)+M S E\right)
$$

where $M S E$, the mean squared error, is calculated as

$$
M S E=\frac{1}{N} \sum_{j=1}^{K} \sum_{\forall z_{p} \in C_{j}} d\left(z_{p}, b i d\right)^{2}
$$

with $N$ indicating the total number of samples (pixels).

\subsection{Proposed algorithm}

Our proposed image clustering algorithm proceeds in the following steps:

Step 1: Parameter initialisation: the parameters include the number of clusters for image segmentation $K$, the number of bids $N$, the number of clusters for bid grouping $K_{H M S}$, the minimum and maximum number of mental searches $M_{l}$ and $M_{H}$. 
Step 2: Create an initial population as

$$
\text { population }=\left[\begin{array}{c}
\text { bid }_{1} \\
\text { bid }_{2} \\
\ldots \\
\operatorname{bid}_{N}
\end{array}\right] \text {, }
$$

where $\operatorname{bid}_{i}=\left[m_{1}, m_{2}, \ldots, m_{K}\right]$

Step 3: Calculate the value of the objective function for each bid.

Step 4: Select the bid with the best objective function value.

Step 5: Select a random number between $M_{l}$ and $M_{h}$ for each bid to define the number of mental searches for each bid.

Step 6: Mental search operator: create new bids in the vicinity of existing bids using a Levy distribution. This operation is carried out using Equations (7) and (9).

Step 7: Replacement operator: in case a new bid is better than a previous one it will replace the latter.

Step 8: Bid grouping: the bid population is clustered using the $k$-means algorithm.

Step 9: Calculate the objective function value for each cluster member.

Step 10: The cluster with the minimum mean objective function value is selected as the winner cluster indicating a promising area.

Step 11: Other bids move toward the best bid available in the winner group based on Equation (12).

Step 12: Find the best bid $b i d^{+}$among all bids and if it is better than the previous best bid bid* replace bid* with it.

Step 13: If the stop condition is not satisfied, go back to Step 3 .

\subsection{An illustrative example}

We illustrate the workings of our proposed algorithm using a simplified example based on the image defined in Figure 3 As the image size is $10 \times 10$, there are 100 samples in the image. Suppose that we want to divide the image into 3 clusters, i.e. $K=3$. In this example, our goal is to minimise the first of the three objective functions, i.e. $C F 1$ as defined in Equation (13). As parameters, we set $N=12, K_{H M S}=3, M_{l}=2$, and $M_{h}=10$.

A randomly created initial population is given in Table 1 
Table 1: Initial population.

\begin{tabular}{lcc}
\hline bid & objective function value & number of mental searches \\
\hline$(120.34,229.38,41.76)$ & 39.26 & 2 \\
$(88.59,127.41,162.08)$ & 49.52 & 5 \\
$(124.51,30.15,222.87)$ & 35.51 & 2 \\
$(109.08,82.61,18.05)$ & 52.09 & 8 \\
$(123.96,188.78,217.79)$ & 50.38 & 4 \\
$(117.61,114.31,197.44)$ & 55.74 & 2 \\
$(36.92,43.58,157.33)$ & 55.32 & 4 \\
$(191.46,190.55,203.70)$ & 58.66 & 3 \\
$(101.29,35.81,15.76)$ & 51.93 & 7 \\
$(41.14,160.28,196.78)$ & 50.64 & 2 \\
$(144.16,47.80,136.06)$ & 56.42 & 5 \\
$(197.55,96.06,146.38)$ & 46.27 & 7 \\
\hline
\end{tabular}

We calculate the CF1 objective function value for the first bid, i.e. for $(120.34,229.38,41.76)$, as

$$
\begin{aligned}
& d_{\max }(Z, b i d)=\max _{j=1, \ldots, K} \sum_{\forall p_{i} \in C_{j}} \frac{d\left(p_{i}, \text { bid }\right)}{\left|C_{j}\right|}=\max (4.04,5.61,3.37)=5.61 \\
& D=\left[\begin{array}{ccc}
\infty & 109.04 & 78.58 \\
109.04 & \infty & 187.62 \\
78.58 & 187.62 & \infty
\end{array}\right] \\
& d_{\min }(\text { bid })=\min _{\forall i, j, i \neq j}\left(d\left(m_{i}, m_{j}\right)\right)=\min (D)=78.58 \\
& J_{e}=\frac{\sum_{j=1}^{K} \sum_{\forall p_{i} \in c_{j}} d\left(p_{i}, \text { bid }\right) /\left|c_{j}\right|}{K}=\frac{4.0398+5.6108+3.3658}{5}=4.34
\end{aligned}
$$

and

$C F 1($ bid,$I)=w_{1} d_{\max }(Z$, bid $)+w_{2}\left(z_{\max }-d_{\min }(I\right.$, bid $\left.)\right)+w_{3} J_{e}=0.4 \times 5.61+0.2 \times(255-78.58)+0.4 \times 4.34=39.26$.

The objective function values for the other bids are calculated equivalently and are listed in Table 1 . From this, we obtain bid ${ }^{*}=(124.51,30.15,222.87)$ as the best bid.

Table 1 also shows the numbers of mental searches assigned for each bid, which were randomly drawn from $\left[M_{l}, M_{h}\right]=$ $[2 ; 10]$.

We next use the mental search operator on the bids. For the first bid, this gives:

$$
\begin{array}{r}
\text { stepsize }=0.01 \times \text { step } \times((120.34,229.38,41.76)-(124.51,30.15,222.87))=(-0.0049,6.9362,-0.5802) \\
\text { newbid }=((120.34,229.38,41.76)+(2-\text { iter } *(2 / \text { max iter })) * \text { stepsize }=(120.33,243.11,40.61)
\end{array}
$$

while another new bid is calculated for the second mental search of this candidate solution in the same fashion and the same operations performed to obtain new bids for all other solutions. 
If an improved bid is found it replaces the previous candidate solution leading to a new set of bids that is listed in Table 2. As can be seen, for all bids the objective function values either improves or stays the same.

Table 2: Population after mental search operations.

\begin{tabular}{lcc}
\hline bid & objective function value & assigned cluster \\
\hline$(120.32,227.14,39.31)$ & 38.55 & 1 \\
$(60.85,127.34,167.64)$ & 47.88 & 3 \\
$(124.51,30.15,222.87)$ & 35.51 & 3 \\
$(109.15,82.59,19.39)$ & 52.05 & 1 \\
$(123.96,177.36,217.78)$ & 48.26 & 3 \\
$(117.58,113.66,197.34)$ & 55.58 & 3 \\
$(36.76,45.37,157.06)$ & 54.76 & 3 \\
$(191.69,190.17,204.04)$ & 58.59 & 2 \\
$(102.50,36.16,227.28)$ & 41.31 & 3 \\
$(58.14,155.05,197.28)$ & 48.79 & 3 \\
$(232.00,45.35,135.09)$ & 37.62 & 2 \\
$(201.04,82.11,146.99)$ & 45.36 & 2 \\
\hline
\end{tabular}

Next, the bids are grouped into $K_{H M S}$ clusters using the $k$-means algorithm. The result of this is also shown in Table 2 where for each bid the resulting cluster number (1,2 or 3 ) is given. As we can see, there are 2 bids in cluster 1,3 bids in cluster 2, and 8 bids in cluster 3. The mean objective function values for the three clusters are 45.30, 47.19, and 47.44, respectively. Yielding the lowest value, cluster 1 is thus the winner cluster.

The best bid in cluster 1 is $(120.32,227.14,39.31)$ with an objective function value of 38.55 . As can be seen, this bid is not the best existing bid as there exists one with an objective function value of 35.51. Finally, the other bids will move toward the winner bid. For example the current bid $(36.76,45.37,157.06)$ is updated as

$(36.76,45.37,157.06)+2 *((0.7,0.3,0.4) \times((120.32,227.14,39.31)-(36.76,45.37,157.06))=(153.74,154.43,62.86)$,

and the other bids are updated in the same fashion.

This concludes the first iteration of the algorithm and the same process will continue until a stopping criterion is met.

\section{Experimental results}

In order to evaluate our proposed image clustering algorithm, we ran an extensive set of experiments. The 11 images that we employed for this purpose, shown in Figure 4, are commonly used for image clustering evaluation: Lenna, House, Airplane, Peppers, MRI and Caspian Sea, and five images from the Berkeley segmentation dataset [34], namely 12003, 42049, 181079, 198054, and 385028.

We compared our proposed algorithm with a number of population-based clustering algorithms that have been previously used for image clustering, including genetic algorithm-based clustering (GA), differential evolution-based clustering (DE), particle swarm optimisation-based clustering (PSO), artificial bee colony algorithm-based clustering (ABC), and harmony search-based clustering (HS), as well as with the $k$-means clustering algorithm.

The population size and the number of iterations for all algorithms were set to 30 and 200, respectively, while we employed the default values for the various parameters which are listed in Table 3 The number of clusters was set to 

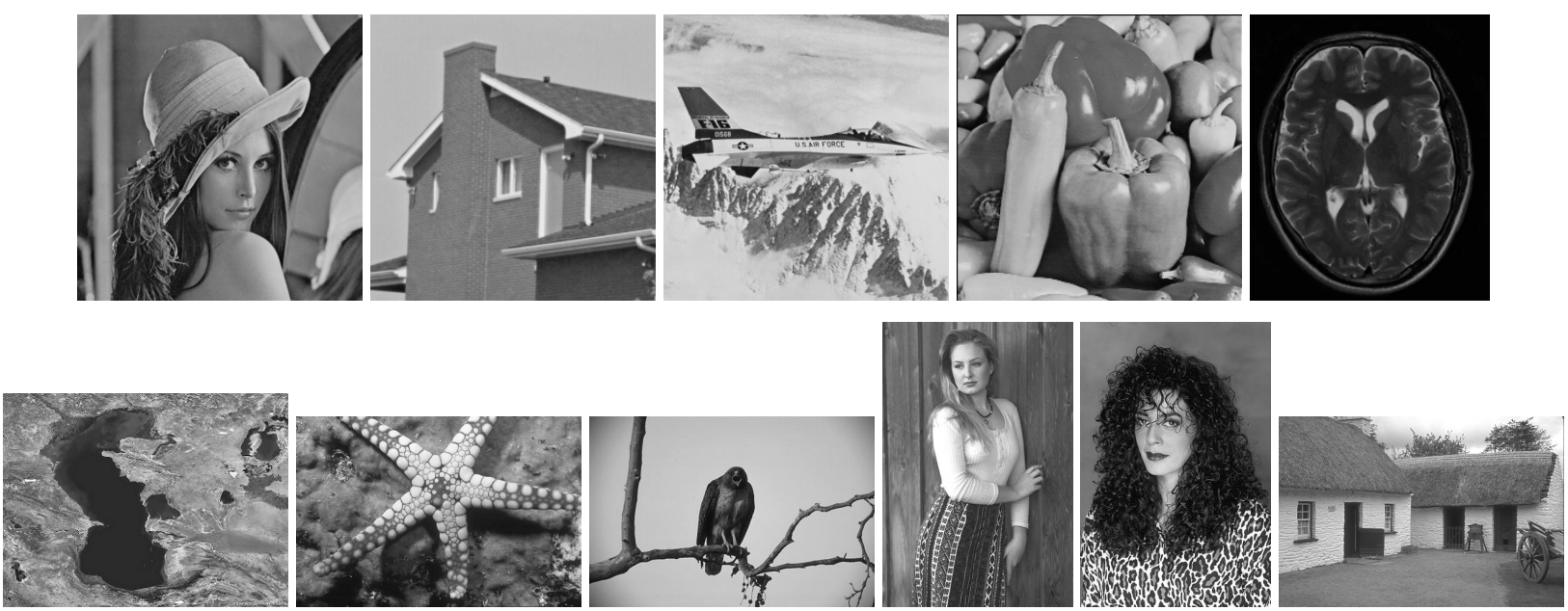

Figure 4: The test image dataset.

5 [14]. Each algorithm was run 50 times and in all instances we report the average results over these 50 runs.

Table 3: Parameter settings for all algorithms.

\begin{tabular}{llc}
\hline algorithm & parameters & value \\
\hline GA & crossover probability & 0.8 \\
& mutation probability & $1 /($ number of thresholds $)$ \\
\hline DE [11] & scaling factor & 0.5 \\
& crossover probability & 0.1 \\
\hline PSO [36] & cognitive constant & 2 \\
& social constant & 2 \\
& inertia constant & 1 to 0 \\
\hline ABC [35] & limit & $n_{e} \times$ dimension of problem \\
\hline HS [13] & harmony memory considering rate & 0.9 \\
& pitch adjusting rate & 0.1 \\
\hline HMS & number of clusters & 5 \\
& C & 2 \\
& &
\end{tabular}

\subsection{Objective functions}

As mentioned above, we are considering three different objective functions. Table 4 gives the obtained results for the three objective functions for all images and algorithms.

As we can see from Table 4, our proposed method consistently leads to better objective function values compared to all other algorithms, confirming its higher efficacy. PSO achieved the best result for Airplane and 12003 based on the CF1 objective function and gives similar CF2 results to HMS for Pepper, Caspian Sean and 42049. ABC shows the best results for House based on CF3, whereas for this objective function PSO yields the best performance for 181079 and 385028 . In all other cases, our proposed HMS image clustering algorithm provides the best objective function results.

We also investigated the convergence speed for all algorithms. Figure 5 shows plots of the objective function values against the number of iterations on the Caspian Sea image (other images showed similar results). As can be seen, our approach leads to faster convergence compared to other algorithms. 
Table 4: Objective function results for all imaged and algorithms. Best results for an image are bolded.

\begin{tabular}{|c|c|c|c|c|c|c|c|}
\hline image & obj. func. & GA & $\mathrm{DE}$ & PSO & $\mathrm{ABC}$ & HS & HMS \\
\hline \multirow[t]{3}{*}{ Lenna } & CF1 & 41.3217 & 40.4814 & 40.0072 & 40.3327 & 41.8099 & 39.4668 \\
\hline & $\mathrm{CF} 2$ & 0.0029 & 0.0029 & 0.0027 & 0.0030 & 0.0035 & 0.0025 \\
\hline & CF3 & 0.0169 & 0.0178 & 0.0154 & 0.0172 & 0.0209 & 0.0151 \\
\hline \multirow[t]{3}{*}{ Airplane } & CF1 & 41.3514 & 40.9192 & 40.3716 & 40.8357 & 42.3934 & 40.3755 \\
\hline & $\mathrm{CF} 2$ & 0.0054 & 0.0053 & 0.0052 & 0.0054 & 0.0060 & 0.0050 \\
\hline & CF3 & 0.0604 & 0.0605 & 0.0572 & 0.0616 & 0.0688 & 0.0564 \\
\hline \multirow[t]{3}{*}{ House } & CF1 & 41.1792 & 40.8318 & 40.2881 & 40.7807 & 42.2414 & 40.283 \\
\hline & $\mathrm{CF} 2$ & 0.0078 & 0.0078 & 0.0080 & 0.0080 & 0.0087 & 0.0072 \\
\hline & CF3 & 0.1229 & 0.1125 & 0.1127 & 0.0080 & 0.1304 & 0.1016 \\
\hline \multirow[t]{3}{*}{ Peppers } & CF1 & 40.5179 & 40.2265 & 39.433 & 40.2053 & 41.2024 & 39.4218 \\
\hline & $\mathrm{CF} 2$ & 0.0061 & 0.0065 & 0.0059 & 0.0068 & 0.0073 & 0.0059 \\
\hline & CF3 & 0.0832 & 0.0879 & 0.0794 & 0.0939 & 0.1082 & 0.0793 \\
\hline \multirow[t]{3}{*}{ MRI } & CF1 & 41.6892 & 41.4657 & 41.0394 & 41.5873 & 42.5158 & 41.0383 \\
\hline & $\mathrm{CF} 2$ & 0.0049 & 0.0049 & 0.0046 & 0.0051 & 0.0055 & 0.0045 \\
\hline & CF3 & 0.0533 & 0.0497 & 0.0458 & 0.0503 & 0.0565 & 0.0439 \\
\hline \multirow[t]{3}{*}{ Caspian Sea } & CF1 & 40.6117 & 39.6926 & 39.1587 & 39.7193 & 40.8176 & 39.0966 \\
\hline & $\mathrm{CF} 2$ & 0.0074 & 0.0075 & 0.0069 & 0.0080 & 0.0082 & 0.0069 \\
\hline & CF3 & 0.1001 & 0.1010 & 0.0945 & 0.1041 & 0.1140 & 0.0903 \\
\hline \multirow[t]{3}{*}{12003} & CF1 & 39.9983 & 39.1849 & 38.5286 & 39.0516 & 40.7451 & 38.5316 \\
\hline & $\mathrm{CF} 2$ & 0.0043 & 0.0044 & 0.0390 & 0.0046 & 0.0050 & 0.0041 \\
\hline & CF3 & 0.0441 & 0.0470 & 4.1915 & 0.0490 & 0.0545 & 0.0041 \\
\hline \multirow[t]{3}{*}{42049} & CF1 & 39.9849 & 39.7542 & 39.1642 & 39.6533 & 41.0621 & 39.1174 \\
\hline & $\mathrm{CF} 2$ & 0.0053 & 0.0054 & 0.0050 & 0.0056 & 0.0063 & 0.0050 \\
\hline & CF3 & 0.0608 & 0.0655 & 0.0614 & 0.0689 & 0.0822 & 0.0587 \\
\hline \multirow[t]{3}{*}{181079} & CF1 & 40.6500 & 40.4703 & 39.6409 & 40.3181 & 39.4609 & 28.1953 \\
\hline & $\mathrm{CF} 2$ & 0.0048 & 0.0050 & 0.0045 & 0.0051 & 0.0055 & 0.0038 \\
\hline & CF3 & 0.0550 & 0.0590 & 0.0515 & 0.0598 & 0.0631 & 0.0578 \\
\hline \multirow[t]{3}{*}{198054} & CF1 & 39.309 & 39.5499 & 38.4583 & 39.0063 & 38.4583 & 28.8709 \\
\hline & $\mathrm{CF} 2$ & 0.0046 & 0.0045 & 0.0043 & 0.0045 & 0.0050 & 0.0032 \\
\hline & CF3 & 0.0562 & 0.0561 & 0.0522 & 0.0550 & 0.0637 & 0.0431 \\
\hline \multirow[t]{3}{*}{385028} & CF1 & 40.2932 & 40.391 & 39.5117 & 40.1178 & 39.5117 & 27.8887 \\
\hline & $\mathrm{CF} 2$ & 0.0040 & 0.0044 & 0.0040 & 0.0045 & 0.0046 & 0.0032 \\
\hline & CF3 & 0.0421 & 0.0475 & 0.0412 & 0.0476 & 0.0508 & 0.0416 \\
\hline
\end{tabular}
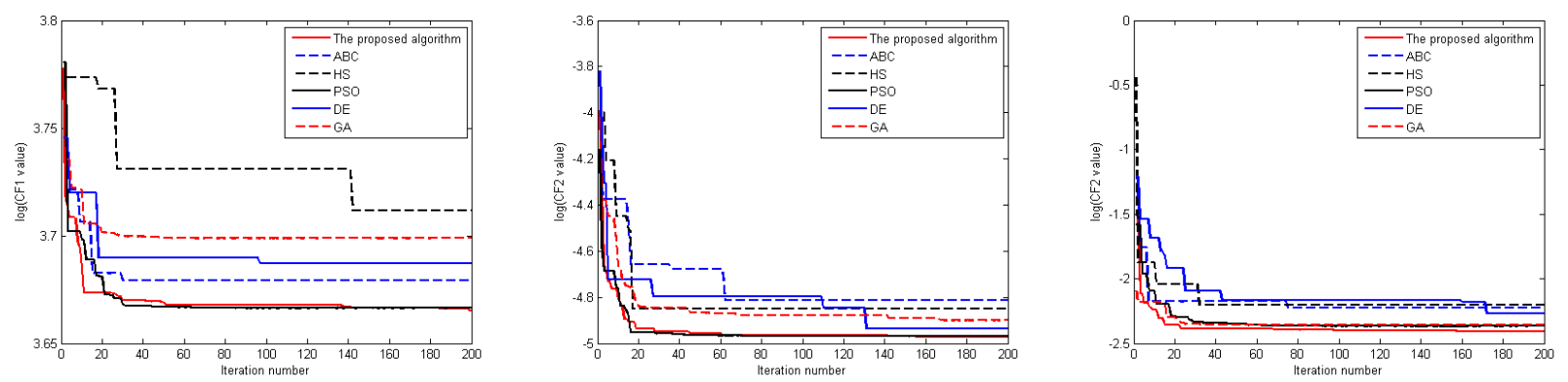

Figure 5: Convergence behaviour of all algorithms on Caspian Sea image for CF1 (left), CF2 (middle) and CF3 (right) objective functions.

\subsection{Clustering validity}

Clustering validity indices are metrics that indicate clustering quality. In general, they assess two aspects of clustering:

1. Compactness: to the extent possible, samples in a cluster should be similar.

2. Separation: clusters should be clearly separated from each other.

In our experiments, we used the Davies-Bouldin (DB) index [37] which is one of the most commonly employed 
clustering validity indices. The DB index measures the proportion of within-cluster scatter to between-cluster separation. The scatter within the $i$-th cluster is calculated as

$$
S_{i}=\frac{1}{N_{i}} \sum_{x_{j} \in c_{i}} d\left(x_{j}, m_{i}\right),
$$

where $N_{i}$ is the number of samples of the $i$-th cluster $c_{i}$, and $d\left(x_{j}, m_{i}\right)$ is the Euclidean distance between sample $x_{j}$ and its cluster centre $m_{i}$. The between-cluster separation is calculated as

$$
R_{i j}=\frac{S_{i}+S_{j}}{d\left(m_{i}, m_{j}\right)}, i \neq j
$$

Finally, the DB index is defined as

$$
D B=\frac{1}{K} \sum_{k=1}^{K} R_{k},
$$

where $R_{k}=\max _{j=1,2, \ldots, K} R_{i j}$ and $i=1,2, \ldots, K$.

Table 5 reports the average DB results over the 50 runs for all three objective functions. As we can see, in 26 out of 33 cases, our proposed approach yields the best (i.e., lowest) DB index.

\subsection{Non-parametric statistical analysis}

Due to the randomness and variance of population-based metaheuristic algorithms, we also conducted a non-parametric statistical analysis. The null hypothesis $H_{0}$ here means that there is no statistical difference between two algorithms, while the alternative hypothesis $H_{1}$ indicates a statistically significant difference between the algorithms. The null hypothesis is the initial statistical claim and if the null hypothesis is rejected the alternative one would be accepted. We utilised two well-known non-parametric tests, namely the Friedman test and the Wilcoxon signed rank test to compare the different image clustering algorithms.

The first stage of the Friedman test is rank calculation for which we list the results in Table 6 As is evident, our proposed algorithm has the lowest average rank with PSO coming second. The chi-squared value and p-value were evaluated as 110.91 and $2.630 \mathrm{e}-22$ respectively. Given the table of chi-squared distribution, the critical value for the degree of freedom (6-1=5) and $\alpha=0.05$ is equal to 11.070 . The chi-squared value is higher which means that $H_{1}$ is accepted. In other words, there is a statistical difference between the algorithms. Moreover, the p-value is extremely small which confirms the rejection of $H_{0}$.

The result (p-value) of the Wilcoxon signed rank test determines the significance level between two algorithms. If the p-value is less than 0.05 , there would be a significant statistical difference between the algorithms. Table 7 reports the obtained results of the Wilcoxon test. As can be seen, there is a significant difference between our proposed algorithm and all other algorithms, with all obtained p-values less than 0.05 . 
Table 5: DB index results for all images and all algorithms. The best result for each image is bolded.

\begin{tabular}{|c|c|c|c|c|c|c|c|}
\hline image & obj. func. & GA & $\mathrm{DE}$ & PSO & $\mathrm{ABC}$ & $\mathrm{HS}$ & HMS \\
\hline \multirow[t]{3}{*}{ Lenna } & CF1 & 0.5511 & 0.5889 & 0.5938 & 0.6383 & 0.5946 & 0.5484 \\
\hline & $\mathrm{CF} 2$ & 0.5371 & 0.5253 & 0.5247 & 0.5266 & 0.5212 & 0.5247 \\
\hline & CF3 & 0.5230 & 0.5257 & 0.5238 & 0.5244 & 0.5239 & 0.5242 \\
\hline \multirow[t]{3}{*}{ Airplane } & CF1 & 0.5556 & 0.5479 & 0.5667 & 0.5634 & 0.5447 & 0.5427 \\
\hline & $\mathrm{CF} 2$ & 0.5396 & 0.536 & 0.5329 & 0.5459 & 0.5212 & 0.5338 \\
\hline & CF3 & 0.5471 & 0.5942 & 0.5597 & 0.6020 & 0.5610 & 0.4942 \\
\hline \multirow[t]{3}{*}{ House } & CF1 & 0.4318 & 0.4286 & 0.4335 & 0.4438 & 0.4587 & 0.4260 \\
\hline & $\mathrm{CF} 2$ & 0.4616 & 0.4522 & 0.4836 & 0.4700 & 0.4840 & 0.4399 \\
\hline & CF3 & 0.5161 & 0.4597 & 0.5048 & 0.4700 & 0.4968 & 0.4463 \\
\hline \multirow[t]{3}{*}{ Peppers } & CF1 & 0.5589 & 0.5555 & 0.5470 & 0.5608 & 0.5679 & 0.5320 \\
\hline & $\mathrm{CF} 2$ & 0.4771 & 0.5062 & 0.4873 & 0.5012 & 0.5119 & 0.4744 \\
\hline & CF3 & 0.4829 & 0.4854 & 0.4949 & 0.4747 & 0.5302 & 0.4641 \\
\hline \multirow[t]{3}{*}{ MRI } & CF1 & 0.5159 & 0.5216 & 0.5159 & 0.5201 & 0.5232 & 0.5144 \\
\hline & $\mathrm{CF} 2$ & 0.4487 & 0.4500 & 0.4493 & 0.4499 & 0.4862 & 0.4421 \\
\hline & CF3 & 0.4793 & 0.4595 & 0.4320 & 0.4462 & 0.4645 & 0.4271 \\
\hline \multirow[t]{3}{*}{ Caspian sea } & CF1 & 0.5440 & 0.5495 & 0.5520 & 0.5411 & 0.5576 & 0.5363 \\
\hline & $\mathrm{CF} 2$ & 0.5575 & 0.5630 & 0.5555 & 0.5546 & 0.5723 & 0.5539 \\
\hline & CF3 & 0.5502 & 0.5562 & 0.5490 & 0.5791 & 0.5471 & 0.5401 \\
\hline \multirow[t]{3}{*}{12003} & CF1 & 0.5483 & 0.5481 & 0.5567 & 0.5475 & 0.5553 & 0.5473 \\
\hline & $\mathrm{CF} 2$ & 0.4953 & 0.4903 & 0.4948 & 0.5116 & 0.5047 & 0.4912 \\
\hline & CF3 & 0.4938 & 0.4986 & 0.4967 & 0.5043 & 0.5342 & 0.4938 \\
\hline \multirow[t]{3}{*}{42049} & CF1 & 0.5415 & 0.5386 & 0.5347 & 0.5420 & 0.5687 & 0.5258 \\
\hline & $\mathrm{CF} 2$ & 0.5017 & 0.5295 & 0.5458 & 0.5079 & 0.5532 & 0.5701 \\
\hline & CF3 & 0.4981 & 0.5148 & 0.4738 & 0.4845 & 0.5591 & 0.4712 \\
\hline \multirow[t]{3}{*}{181079} & CF1 & 0.5861 & 0.5758 & 0.5723 & 0.5725 & 0.5711 & 0.5675 \\
\hline & $\mathrm{CF} 2$ & 0.5531 & 0.5578 & 0.5578 & 0.5534 & 0.5520 & 0.5418 \\
\hline & CF3 & 0.5091 & 0.5092 & 0.5079 & 0.5006 & 0.5088 & 0.5085 \\
\hline \multirow[t]{3}{*}{198054} & CF1 & 0.4885 & 0.4865 & 0.4800 & 0.4831 & 0.4941 & 0.4793 \\
\hline & $\mathrm{CF} 2$ & 0.4757 & 0.4450 & 0.4566 & 0.4777 & 0.4759 & 0.4327 \\
\hline & CF3 & 0.4552 & 0.4671 & 0.4509 & 0.4425 & 0.4710 & 0.4390 \\
\hline \multirow[t]{3}{*}{385028} & CF1 & 0.5471 & 0.5478 & 0.5420 & 0.5457 & 0.5403 & 0.5483 \\
\hline & $\mathrm{CF} 2$ & 0.5998 & 0.5755 & 0.5704 & 0.5700 & 0.5957 & 0.5649 \\
\hline & CF3 & 0.5248 & 0.5013 & 0.5038 & 0.5187 & 0.5081 & 0.5006 \\
\hline
\end{tabular}

Table 6: Ranks for all algorithms.

\begin{tabular}{lc}
\hline algorithm & average rank \\
\hline GA & 3.8636 \\
DE & 3.8788 \\
PSO & 2.3182 \\
ABC & 4.1061 \\
HS & 5.6061 \\
HMS & 1.2273 \\
\hline
\end{tabular}

Table 7: Results of Wilcoxon signed rank test.

\begin{tabular}{lc}
\hline & p-value \\
\hline HMS vs. GA & $2.0905 \mathrm{e}-06$ \\
HMS vs. DE & $5.3525 \mathrm{e}-05$ \\
HMS vs. PSO & $2.8234 \mathrm{e}-04$ \\
HMS vs. ABC & $3.8488 \mathrm{e}-06$ \\
HMS vs. HS & $5.3720 \mathrm{e}-07$ \\
\hline
\end{tabular}


Table 8: Standard deviations for all images and algorithms. The best result for each image is bolded.

\begin{tabular}{|c|c|c|c|c|c|c|c|}
\hline image & obj. func. & GA & $\mathrm{DE}$ & PSO & $\mathrm{ABC}$ & $\mathrm{HS}$ & HMS \\
\hline \multirow[t]{3}{*}{ Lenna } & CF1 & $7.61 \mathrm{e}-01$ & $2.45 \mathrm{e}-01$ & $1.30 \mathrm{e}-01$ & $1.98 \mathrm{e}-01$ & $4.03 \mathrm{e}-01$ & $1.82 \mathrm{e}-07$ \\
\hline & $\mathrm{CF} 2$ & $1.14 \mathrm{e}-04$ & $7.54 \mathrm{e}-05$ & $1.17 \mathrm{e}-05$ & $1.07 \mathrm{e}-04$ & $2.04 \mathrm{e}-04$ & 4.38e-07 \\
\hline & CF3 & $1.69 \mathrm{e}-03$ & $6.73 e-04$ & $7.67 e-05$ & $1.12 \mathrm{e}-03$ & $2.45 \mathrm{e}-03$ & $5.13 e-06$ \\
\hline \multirow[t]{3}{*}{ Airplane } & CF1 & $3.86 \mathrm{e}-01$ & $2.84 \mathrm{e}-01$ & $2.44 \mathrm{e}-04$ & $1.39 \mathrm{e}-01$ & $5.83 \mathrm{e}-01$ & $1.53 \mathrm{e}-03$ \\
\hline & $\mathrm{CF} 2$ & $2.16 \mathrm{e}-04$ & $1.19 \mathrm{e}-04$ & $2.55 \mathrm{e}-04$ & $1.00 \mathrm{e}-04$ & $3.44 \mathrm{e}-04$ & $8.13 \mathrm{e}-06$ \\
\hline & CF3 & $2.35 \mathrm{e}-03$ & $8.72 \mathrm{e}-04$ & $4.84 \mathrm{e}-04$ & $1.25 \mathrm{e}-03$ & $4.21 \mathrm{e}-03$ & $1.31 \mathrm{e}-04$ \\
\hline \multirow[t]{3}{*}{ House } & CF1 & $6.37 \mathrm{e}-01$ & $3.01 \mathrm{e}-01$ & $1.87 \mathrm{e}-02$ & $1.70 \mathrm{e}-01$ & $5.50 \mathrm{e}-01$ & $2.69 \mathrm{e}-03$ \\
\hline & $\mathrm{CF} 2$ & $6.43 e-04$ & $2.22 \mathrm{e}-04$ & $4.65 e-04$ & $2.94 \mathrm{e}-04$ & $5.57 \mathrm{e}-04$ & $1.60 \mathrm{e}-05$ \\
\hline & CF3 & $8.05 \mathrm{e}-03$ & $7.07 \mathrm{e}-03$ & $1.05 \mathrm{e}-02$ & $2.94 \mathrm{e}-04$ & $1.12 \mathrm{e}-02$ & $5.72 \mathrm{e}-04$ \\
\hline \multirow[t]{3}{*}{ Peppers } & CF1 & $7.27 \mathrm{e}-01$ & $2.61 \mathrm{e}-01$ & $2.46 \mathrm{e}-02$ & $2.46 \mathrm{e}-01$ & $4.32 \mathrm{e}-01$ & $4.48 \mathrm{e}-03$ \\
\hline & $\mathrm{CF} 2$ & $2.41 \mathrm{e}-04$ & $1.90 \mathrm{e}-04$ & $6.31 \mathrm{e}-05$ & $3.47 \mathrm{e}-04$ & $5.08 \mathrm{e}-04$ & $5.81 \mathrm{e}-06$ \\
\hline & CF3 & $2.44 \mathrm{e}-03$ & $2.89 \mathrm{e}-03$ & $3.86 \mathrm{e}-04$ & $8.24 \mathrm{e}-03$ & $1.17 \mathrm{e}-02$ & $1.08 \mathrm{e}-04$ \\
\hline \multirow[t]{3}{*}{ MRI } & CF1 & $5.75 e-01$ & $2.09 \mathrm{e}-01$ & $2.27 \mathrm{e}-03$ & $1.24 \mathrm{e}-01$ & $4.39 \mathrm{e}-01$ & $1.17 \mathrm{e}-03$ \\
\hline & $\mathrm{CF} 2$ & $3.00 \mathrm{e}-04$ & $1.50 \mathrm{e}-04$ & $4.75 e-05$ & $1.47 \mathrm{e}-04$ & $2.33 \mathrm{e}-04$ & $1.86 \mathrm{e}-06$ \\
\hline & CF3 & $8.31 \mathrm{e}-03$ & $1.67 \mathrm{e}-03$ & $3.42 \mathrm{e}-04$ & $3.24 \mathrm{e}-03$ & $3.05 \mathrm{e}-03$ & $5.02 \mathrm{e}-05$ \\
\hline \multirow[t]{3}{*}{ Caspian sea } & CF1 & $3.02 \mathrm{e}-01$ & $2.67 e-01$ & $8.93 e-02$ & $2.14 \mathrm{e}-01$ & $3.98 \mathrm{e}-01$ & $1.61 e-03$ \\
\hline & $\mathrm{CF} 2$ & $3.62 \mathrm{e}-04$ & $1.75 \mathrm{e}-04$ & $4.09 \mathrm{e}-05$ & $3.69 \mathrm{e}-04$ & $4.40 \mathrm{e}-04$ & 1.01e-05 \\
\hline & CF3 & $4.20 \mathrm{e}-03$ & $1.74 \mathrm{e}-03$ & $9.27 \mathrm{e}-04$ & $3.53 \mathrm{e}-03$ & $2.32 \mathrm{e}-03$ & $1.97 \mathrm{e}-04$ \\
\hline \multirow[t]{3}{*}{12003} & CF1 & $6.07 \mathrm{e}-01$ & $2.51 \mathrm{e}-01$ & $6.37 \mathrm{e}-04$ & $1.43 \mathrm{e}-01$ & $8.29 \mathrm{e}-01$ & $2.17 \mathrm{e}-03$ \\
\hline & $\mathrm{CF} 2$ & $2.71 \mathrm{e}-04$ & $1.20 \mathrm{e}-04$ & $2.95 \mathrm{e}-04$ & $2.24 \mathrm{e}-04$ & $2.54 \mathrm{e}-04$ & $6.17 \mathrm{e}-06$ \\
\hline & CF3 & $3.20 \mathrm{e}-03$ & $1.88 \mathrm{e}-03$ & $5.52 \mathrm{e}-02$ & $2.36 \mathrm{e}-03$ & $3.50 \mathrm{e}-03$ & $4.99 \mathrm{e}-06$ \\
\hline \multirow[t]{3}{*}{42049} & CF1 & $4.46 \mathrm{e}-01$ & $2.78 \mathrm{e}-01$ & $7.92 \mathrm{e}-02$ & $2.32 \mathrm{e}-01$ & $5.88 \mathrm{e}-01$ & $3.73 e-03$ \\
\hline & $\mathrm{CF} 2$ & $2.73 \mathrm{e}-04$ & $1.34 \mathrm{e}-04$ & $3.40 \mathrm{e}-05$ & $2.42 \mathrm{e}-04$ & $4.71 \mathrm{e}-04$ & $7.14 \mathrm{e}-06$ \\
\hline & CF3 & $1.50 \mathrm{e}-03$ & $2.06 \mathrm{e}-03$ & $7.41 \mathrm{e}-04$ & $5.14 \mathrm{e}-03$ & $6.29 \mathrm{e}-03$ & $1.52 \mathrm{e}-04$ \\
\hline \multirow[t]{3}{*}{181079} & CF1 & $5.79 \mathrm{e}-01$ & $2.45 \mathrm{e}-01$ & $4.32 \mathrm{e}-05$ & $1.45 \mathrm{e}-01$ & $5.88 \mathrm{e}-01$ & $1.58 \mathrm{e}-04$ \\
\hline & CF2 & $2.49 \mathrm{e}-04$ & $2.74 \mathrm{e}-04$ & $4.77 e-05$ & $1.84 \mathrm{e}-04$ & $3.49 \mathrm{e}-04$ & $1.79 \mathrm{e}-06$ \\
\hline & CF3 & $4.80 \mathrm{e}-03$ & $3.78 \mathrm{e}-03$ & $9.57 e-04$ & $2.53 \mathrm{e}-03$ & $7.17 \mathrm{e}-03$ & $5.59 \mathrm{e}-05$ \\
\hline \multirow[t]{3}{*}{198054} & CF1 & $6.60 \mathrm{e}-01$ & $3.60 \mathrm{e}-01$ & $2.60 \mathrm{e}-04$ & $3.70 \mathrm{e}-01$ & $5.97 \mathrm{e}-01$ & 8.47e-05 \\
\hline & $\mathrm{CF} 2$ & $2.78 \mathrm{e}-04$ & $7.51 \mathrm{e}-05$ & $1.81 \mathrm{e}-04$ & $8.38 \mathrm{e}-05$ & $3.17 \mathrm{e}-04$ & $1.84 \mathrm{e}-06$ \\
\hline & CF3 & $5.67 \mathrm{e}-03$ & $1.61 \mathrm{e}-03$ & $2.27 \mathrm{e}-03$ & $2.26 \mathrm{e}-03$ & $4.79 \mathrm{e}-03$ & $6.49 \mathrm{e}-05$ \\
\hline \multirow[t]{3}{*}{385028} & CF1 & $5.17 \mathrm{e}-01$ & $4.12 \mathrm{e}-01$ & $5.35 e-04$ & $3.25 \mathrm{e}-01$ & $4.40 \mathrm{e}-01$ & $5.44 \mathrm{e}-05$ \\
\hline & $\mathrm{CF} 2$ & $5.86 \mathrm{e}-05$ & $2.47 \mathrm{e}-04$ & $2.62 \mathrm{e}-04$ & $1.76 \mathrm{e}-04$ & $3.57 \mathrm{e}-04$ & $1.45 \mathrm{e}-06$ \\
\hline & CF3 & $3.10 \mathrm{e}-03$ & $3.17 \mathrm{e}-03$ & $3.73 e-03$ & $2.77 \mathrm{e}-03$ & $4.13 \mathrm{e}-03$ & $7.72 \mathrm{e}-05$ \\
\hline
\end{tabular}

\subsection{Stability analysis}

Due to the randomness of metaheuristic algorithms, each run of an algorithm will generate a different result. A lower dispersion among multiple runs of an algorithm hence indicates a more stable algorithm. We therefore analyse the stability of the evaluated techniques and employ the standard deviation

$$
S T D=\sqrt{\sum_{i=1}^{M} \frac{\left(x_{i}-\mu\right)^{2}}{M}},
$$

where $M$ is the number of runs ( $M=50$ in our experiments), $x_{i}$ is the objective function value obtained for the $i$-th run and $\mu$ is the mean objective function value for the algorithm.

Table 8 lists the obtained results. As can be seen, in all but 4 cases, our proposed method yields the lowest standard deviation, which demonstrates that our approach is more stable in comparison with other metaheuristic-based image clustering techniques. 


\subsection{Comparison with conventional clustering-based methods}

In addition to comparing our proposed HMS-based algorithm to image clustering based on other metaheuristic optimisationbased approaches, we also compared its performance to conventional clustering-based methods, namely $k$-means, fuzzy $c$-means, and Gaussian mixture model (GMM). We perform this, employing the CF3 objective function for HMS and using the DB index as evaluation criterion. The results are given in Table 9 which shows that our approach clearly outperforms the other algorithms in most cases. We also performed a Wilcoxon signed rank test to compare the methods; Table 10 confirms that our HMS algorithm is statistically significantly better than the other approaches.

Table 9: DB index results of conventional clustering-based methods and HMS.

\begin{tabular}{llccc}
\hline image & $k$-means & fuzzy $c$-means & GMM & HMS \\
\hline Lenna & 0.5427 & 0.5151 & 0.5468 & 0.5242 \\
Airplane & 0.5395 & 0.5198 & 0.5983 & 0.4942 \\
House & 0.4613 & 0.4967 & 0.9548 & 0.4463 \\
Peppers & 0.4984 & 0.4922 & 0.5959 & 0.4641 \\
MRI & 0.4639 & 0.4574 & 0.5165 & 0.4271 \\
Caspian sea & 0.5442 & 0.5240 & 0.8163 & 0.5401 \\
12003 & 0.5001 & 0.4987 & 0.5559 & 0.4938 \\
42049 & 0.5674 & 0.5124 & 0.6493 & 0.4712 \\
181079 & 0.5435 & 0.5162 & 0.6248 & 0.5085 \\
198054 & 0.4798 & 0.4437 & 0.5119 & 0.4390 \\
385028 & 0.5287 & 0.5182 & 0.5374 & 0.5006 \\
\hline
\end{tabular}

Table 10: Results of Wilcoxon signed rank test between HMS and conventional clustering-based methods.

\begin{tabular}{ll}
\hline algorithms & p-value \\
\hline HMS vs. $k$-means & $9.7656 \mathrm{e}-04$ \\
HMS vs. fuzzy $c$-means & 0.0322 \\
HMS vs. GMM & $9.7656 \mathrm{e}-04$ \\
\hline
\end{tabular}

\subsection{Search ability in high dimensions}

The curse of dimensionality is a well-known problem that can occur when employing optimisation-based algorithms, since increasing the dimensionality of a problem exponentially expands the search space. In our image clustering problem, dimensionality primarily relates to the number of clusters (image regions) for which the algorithm tries to identify the best configuration.

In Table 11 we hence report results with different numbers of clusters, namely $K=\{5,10,15\}$. As can be seen from there, under all settings our proposed algorithm outperforms all other approaches. Only in 5 out of the 33 cases, one of the other algorithms gives a better clustering result, while this happens only once each for the more difficult cases of $K=10$ and $K=15$ indicating that our approach scales better with regards to the dimensionality of the problem compared to other techniques.

The superiority of our approach is also confirmed in Tables 12 and 13 which give the results of the Friedman and Wilcoxon test for $K=10$ and $K=15$ respectively. Both tests confirm that the proposed HMS algorithm performs statistically significantly better than the other metaheuristics (with the exception of HMS vs. PSO for $K=10$ ). 
Table 11: Obtained results for different numbers of clusters. The best results for each case are bolded.

\begin{tabular}{lccccccc}
\hline image & $K$ & GA & DE & PSO & ABC & HS & HMS \\
\hline Lenna & 5 & 0.0169 & 0.0178 & 0.0154 & 0.0172 & 0.0209 & $\mathbf{0 . 0 1 5 1}$ \\
& 10 & 0.0271 & 0.0323 & 0.0353 & 0.0617 & 0.0619 & $\mathbf{0 . 0 2 2 2}$ \\
& 15 & 0.0349 & 0.0548 & 0.0463 & 0.1212 & 0.1087 & $\mathbf{0 . 0 3 4 8}$ \\
\hline Airplane & 5 & 0.0604 & 0.0605 & 0.0572 & 0.0616 & 0.0688 & $\mathbf{0 . 0 5 6 4}$ \\
& 10 & 0.0894 & 0.1110 & 0.1310 & 0.1204 & 0.1486 & $\mathbf{0 . 0 7 7 5}$ \\
& 15 & 0.1239 & 0.1793 & 0.1107 & 0.2692 & 0.2688 & $\mathbf{0 . 0 9 7 4}$ \\
\hline House & 5 & 0.1229 & 0.1125 & 0.1127 & $\mathbf{0 . 0 0 8 0}$ & 0.1304 & 0.1016 \\
& 10 & 0.2981 & 0.3062 & 0.2780 & 0.5316 & 0.4275 & $\mathbf{0 . 2 0 2 3}$ \\
& 15 & 0.3400 & 0.6739 & 0.4209 & 1.0115 & 1.0126 & $\mathbf{0 . 3 2 6 0}$ \\
\hline Peppers & 5 & 0.0832 & 0.0879 & 0.0794 & 0.0939 & 0.1082 & $\mathbf{0 . 0 7 9 3}$ \\
& 10 & 0.1564 & 0.1664 & 0.1292 & 0.2140 & 0.2505 & $\mathbf{0 . 1 1 0 4}$ \\
& 15 & 0.3665 & 0.2109 & 0.2998 & 0.4263 & 0.4255 & $\mathbf{0 . 1 3 6 3}$ \\
\hline MRI & 5 & 0.0533 & 0.0497 & 0.0458 & 0.0503 & 0.0565 & $\mathbf{0 . 0 4 3 9}$ \\
& 10 & 0.0831 & 0.1064 & 0.0658 & 0.1227 & 0.1416 & $\mathbf{0 . 0 6 5 7}$ \\
& 15 & 0.1604 & 0.1431 & 0.1575 & 0.2851 & 0.2785 & $\mathbf{0 . 0 8 1 7}$ \\
\hline Caspian sea & 5 & 0.1001 & 0.1010 & 0.0945 & 0.1041 & 0.1140 & $\mathbf{0 . 0 9 0 3}$ \\
& 10 & 0.2886 & 0.2562 & 0.2745 & 0.3417 & 0.4807 & $\mathbf{0 . 2 0 4 6}$ \\
& 15 & 0.5903 & 1.0795 & $\mathbf{0 . 4 8 2 8}$ & 2.0182 & 2.0531 & 0.6128 \\
\hline 12003 & 5 & 0.0441 & 0.0470 & 4.1915 & 0.0490 & 0.0545 & $\mathbf{0 . 0 0 4 1}$ \\
& 10 & 0.0791 & 0.0844 & 0.0658 & 0.1516 & 0.1207 & $\mathbf{0 . 0 5 9 0}$ \\
& 15 & 0.1229 & 0.1499 & 0.1095 & 0.2627 & 0.2025 & $\mathbf{0 . 0 8 3 0}$ \\
\hline 42049 & 5 & 0.0608 & 0.0655 & 0.0614 & 0.0689 & 0.0822 & $\mathbf{0 . 0 5 8 7}$ \\
& 10 & 0.0985 & 0.1244 & 0.0853 & 0.1617 & 0.1390 & $\mathbf{0 . 0 7 8 3}$ \\
& 15 & 0.1426 & 0.1739 & 0.1414 & 0.4123 & 0.4626 & $\mathbf{0 . 0 9 9 0}$ \\
\hline 58054 & 10 & 0.0896 & 0.1250 & 0.0956 & 0.1764 & 0.1756 & $\mathbf{0 . 0 8 7 5}$ \\
& 15 & 0.1494 & 0.1594 & 0.1423 & 0.1875 & 0.2710 & $\mathbf{0 . 1 1 7 9}$ \\
\hline & 10 & 0.0562 & 0.0561 & 0.0522 & 0.0550 & 0.0637 & $\mathbf{0 . 0 4 3 1}$ \\
& 15 & 0.1131 & 0.1517 & 0.0974 & 0.1847 & 0.1767 & $\mathbf{0 . 0 9 8 2}$ \\
& 5 & 0.0421 & 0.0475 & $\mathbf{0 . 0 4 1 2}$ & 0.0476 & 0.0508 & 0.0416 \\
& 10 & 0.0626 & 0.0900 & $\mathbf{0 . 0 5 9 9}$ & 0.0989 & 0.1125 & $\mathbf{0 . 0 5 9 9}$ \\
& 0.1143 & 0.1430 & 0.0871 & 0.2491 & 0.2139 & $\mathbf{0 . 0 1 1 4}$ \\
\hline & & & & & &
\end{tabular}

Table 12: Results of Friedman tests for $K=10$ and $K=15$

\begin{tabular}{lcc}
\hline algorithm & average rank $K=10$ & average rank $K=15$ \\
\hline GA & 2.82 & 2.91 \\
DE & 3.64 & 3.64 \\
PSO & 2.50 & 2.27 \\
ABC & 5.36 & 5.55 \\
HS & 5.55 & 5.45 \\
HMS & 1.13 & 1.18 \\
\hline \hline chi-squared & 33.79 & 47.94 \\
p-value & $7.5068 \mathrm{e}-09$ & $3.6620 \mathrm{e}-09$ \\
\hline
\end{tabular}

Table 13: Results of Wilcoxon signed rank tests for $K=10$ and $K=15$.

\begin{tabular}{lcc}
\hline & p-value $K=10$ & p-value $K=15$ \\
\hline HMS vs. GA & $9.7565 \mathrm{e}-04$ & 0.0049 \\
HMS vs. DE & $9.7565 \mathrm{e}-04$ & $9.7565 \mathrm{e}-04$ \\
HMS vs. PSO & 0.0059 & 0.0420 \\
HMS vs. ABC & $9.7565 \mathrm{e}-04$ & $9.7565 \mathrm{e}-04$ \\
HMS vs. HS & $9.7565 \mathrm{e}-04$ & $9.7565 \mathrm{e}-04$ \\
\hline
\end{tabular}




\subsection{Parameter settings}

Like every metaheuristic algorithm, HMS also has some parameters which in turn affect the performance of the algorithm. While in the experiments above, we have selected default values for the HMS parameters, in our next experiment we investigated the behaviour of our algorithm with respect to the two main parameters of the algorithm, namely the number of clusters for bid grouping $K_{H M S}$, and the number of mental searches.

Our analysis is inspired by [32,38], and we selected two images, House and Peppers, and the CF3 objective function for this purpose. The House image was chosen as here, for the CF3 function, HMS did not provide the best result, while for Peppers our approach yielded the best performance.

\subsubsection{Number of clusters for grouping bids}

As mentioned in [32], more local optima might require a higher number of clusters. Figure 6 illustrates the effect of the number of clusters on the mean objective function value. As can be seen, by increasing the number of clusters for the House image, the mean objective function value decreases, while similar results, though on a smaller scale, are observed for the Pepper image. This indicates that the number of clusters can have a considerable effect on the efficacy of the algorithm and lead to improved clustering performance.
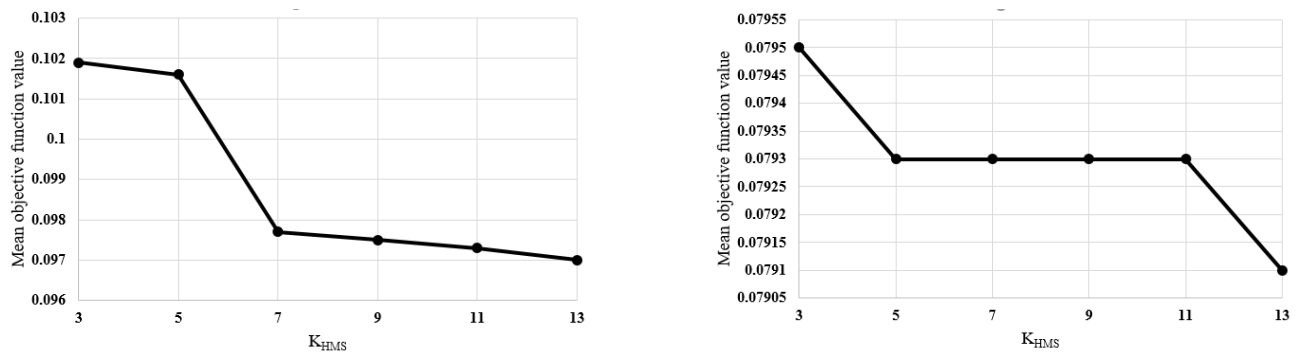

Figure 6: Number of clusters for bid grouping vs. mean objective function value for House (left) and Peppers (right)

\subsubsection{Number of mental searches}

As mentioned, the number of mental searches is randomly selected between the user-defined lower $(L)$ and upper $(H)$ limit. We therefore asses the algorithm's sensitivity to different settings of this interval. The results are given in Figure 7 As can be observed from there, an increased number of mental searches will in general lead to better image clustering performance.
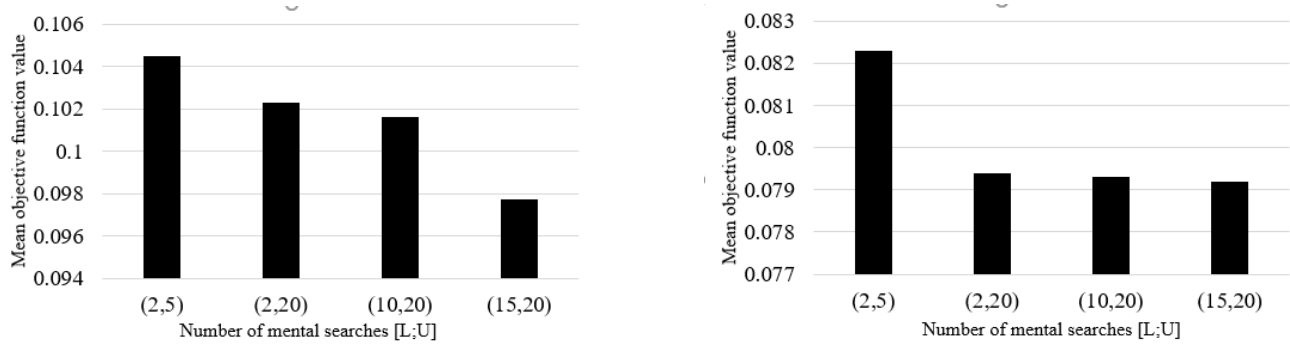

Figure 7: Effect of the number of mental searches on the mean objective function value for House (left) and Peppers (right) 


\subsection{Image segmentation performance}

Last not least, we evaluated the performance of our proposed algorithm in terms of image segmentation quality. Clearly this is crucial, since image clustering is primarily employed for the purpose of segmentation. For this, we used three unsupervised and two supervised image segmentation criteria from the literature. While unsupervised criteria do not require a manually-segmented ground truth images, this is necessary for supervised criteria. As only the images of the Berkeley segmentation dataset come with such a ground truth, the experiments for supervised criteria were conducted only on these images, i.e. images 12003, 42049, 181079, 198054, and 385028.

The Borsotti criterion (BOR) [39] is an unsupervised segmentation metric derived from the number, the variance, and the area of segmented image regions, with a lower BOR indicating better segmentation performance. Tables 14 shows the BOR results for all algorithms on the test image set. As we can see, for 8 of the 11 images, our HMS approach affords the best BOR results. This is further confirmed in the calculated rankings of the algorithms, which is also given in Table 14.

Table 14: BOR results for all images and all algorithms. Best results are bolded.

\begin{tabular}{lcccccc}
\hline image & GA & DE & PSO & ABC & HS & HMS \\
\hline Lenna & 0.0210 & 0.0204 & 0.0197 & 0.0205 & 0.0220 & $\mathbf{0 . 0 1 9 6}$ \\
Airplane & 0.0208 & 0.0204 & 0.0196 & 0.0205 & 0.0196 & $\mathbf{0 . 0 1 9 0}$ \\
House & 0.0188 & 0.0174 & 0.0182 & 0.0197 & 0.0191 & $\mathbf{0 . 0 1 6 9}$ \\
Peppers & 0.0282 & 0.0287 & $\mathbf{0 . 0 2 7 4}$ & 0.0300 & 0.0301 & 0.0275 \\
MRI & 0.0158 & 0.0130 & 0.0130 & 0.0132 & 0.0142 & $\mathbf{0 . 0 1 2 8}$ \\
Caspian Sea & 0.0299 & 0.0304 & $\mathbf{0 . 0 2 8 6}$ & 0.0291 & 0.0301 & 0.0292 \\
12003 & 0.0359 & $\mathbf{0 . 0 3 5 8}$ & 0.0360 & 0.0362 & 0.0377 & $\mathbf{0 . 0 3 5 8}$ \\
42049 & 0.0149 & 0.0147 & 0.0141 & 0.0152 & 0.0160 & $\mathbf{0 . 0 1 4 0}$ \\
181079 & 0.0561 & 0.0571 & $\mathbf{0 . 0 5 4 8}$ & 0.056 & 0.0554 & 0.0551 \\
198054 & 0.0710 & 0.0761 & 0.0704 & 0.0692 & 0.0704 & $\mathbf{0 . 0 6 9 1}$ \\
385028 & 0.0674 & 0.0679 & 0.0670 & 0.0671 & 0.0677 & $\mathbf{0 . 0 6 6 3}$ \\
\hline average rank & 4.45 & 4.00 & 2.23 & 4.09 & 4.82 & 1.41 \\
\hline
\end{tabular}

Another unsupervised criterion used to evaluate image segmentation performance is Levine and Nazif's interclass contrast (LNIC) [40]. This criterion calculates the sum of region contrasts weighted by their areas, with a higher LNIC value signifying better performance. Table 15 summarises the obtained LNIC results for all algorithms. As we can see, our proposed approach yields the best result in 9 of the 11 cases and is clearly ranked first among the 6 approaches.

Table 15: LNIC results for all images and all algorithms. Best results are bolded.

\begin{tabular}{lcccccc}
\hline image & GA & DE & PSO & ABC & HS & HMS \\
\hline Lenna & 0.2391 & 0.2368 & 0.2368 & 0.2381 & 0.2405 & $\mathbf{0 . 2 5 9 6}$ \\
Airplane & 0.1241 & 0.1207 & 0.1229 & 0.1242 & 0.1211 & $\mathbf{0 . 1 2 4 3}$ \\
House & 0.1715 & $\mathbf{0 . 1 8 3 4}$ & 0.1731 & 0.1703 & 0.1743 & 0.1785 \\
Peppers & 0.2592 & 0.2590 & 0.2597 & $\mathbf{0 . 2 6 0 9}$ & 0.2598 & 0.2599 \\
MRI & 0.6202 & 0.6260 & 0.6254 & 0.6226 & 0.6236 & $\mathbf{0 . 6 2 8 3}$ \\
Caspian Sea & 0.2134 & 0.2131 & 0.2132 & 0.2122 & 0.2120 & $\mathbf{0 . 2 1 3 9}$ \\
12003 & 0.2607 & 0.2607 & 0.2602 & 0.2606 & 0.2591 & $\mathbf{0 . 2 6 1 6}$ \\
42049 & 0.2492 & 0.2505 & 0.2513 & 0.2411 & 0.2531 & $\mathbf{0 . 2 5 7 7}$ \\
181079 & 0.2343 & 0.2437 & 0.2344 & 0.2387 & 0.2401 & $\mathbf{0 . 2 8 8 5}$ \\
198054 & 0.4381 & 0.4345 & 0.4319 & 0.4310 & 0.4376 & $\mathbf{0 . 5 2 0 7}$ \\
385028 & 0.2022 & 0.2032 & 0.2028 & 0.2060 & 0.2048 & $\mathbf{0 . 2 5 7 2}$ \\
\hline average rank & 4.14 & 3.73 & 4.23 & 4.09 & 3.64 & 1.18 \\
\hline
\end{tabular}

Levine and Nazif also proposed an intra-class uniformity criterion (LNIU) [40], which is based on the sum of nor- 
malised standard deviations of the image regions. A higher LNIU value indicates better segmentation performance. For 8 of the 11 images, the proposed HMS algorithm achieves the best LNIU result and is thus also ranked first overall.

Table 16: LNIU results for all images and all algorithms. Best results are bolded.

\begin{tabular}{lcccccc}
\hline image & GA & DE & PSO & ABC & HS & HMS \\
\hline Lenna & 0.0329 & 0.0323 & 0.0314 & 0.0322 & 0.0338 & $\mathbf{0 . 0 5 8 6}$ \\
Airplane & 0.0333 & 0.0354 & 0.0341 & 0.0343 & 0.0335 & $\mathbf{0 . 0 3 9 2}$ \\
House & 0.0228 & $\mathbf{0 . 0 2 6 5}$ & 0.0233 & 0.0236 & 0.0233 & 0.0256 \\
Peppers & 0.0395 & $\mathbf{0 . 0 3 9 9}$ & 0.0398 & 0.0392 & 0.0392 & $\mathbf{0 . 0 3 9 9}$ \\
42049 & 0.0353 & 0.0356 & 0.0346 & 0.0359 & $\mathbf{0 . 0 3 6 4}$ & 0.0359 \\
12003 & 0.0526 & 0.0529 & 0.0521 & 0.0534 & 0.0534 & $\mathbf{0 . 0 5 3 5}$ \\
181079 & 0.0486 & 0.0506 & 0.0489 & 0.0497 & 0.0489 & $\mathbf{0 . 0 8 1 9}$ \\
198054 & 0.0592 & 0.0550 & 0.0591 & 0.0552 & 0.0578 & $\mathbf{0 . 0 9 3 6}$ \\
385028 & 0.0515 & 0.0501 & 0.0506 & 0.0506 & 0.0519 & $\mathbf{0 . 0 8 2 0}$ \\
MRI & 0.0322 & 0.0321 & 0.0321 & 0.0320 & $\mathbf{0 . 0 3 2 5}$ & 0.0324 \\
Caspian Sea & 0.0384 & $\mathbf{0 . 0 3 9 0}$ & 0.0389 & 0.0388 & 0.0388 & $\mathbf{0 . 0 3 9 0}$ \\
\hline average rank & 4.46 & 3.32 & 4.46 & 4.05 & 3.32 & 1.41 \\
\hline
\end{tabular}

The two supervised criteria we evaluated are the variation of information and the probabilistic Rand index. The variation of information ( $\mathrm{VoI})$ [41] computes the information shared between a ground truth and an automatic segmentation by calculating the information that is lost or gained when changing from one to the other. A lower VoI value relates to a better segmentation. Table 17 lists the VoI results for the tested Berkeley images and all algorithms. Impressively, for all five images our proposed algorithm gives the best result.

Table 17: VoI results for Berkeley images and all algorithms. Best results are bolded.

\begin{tabular}{lcccccc}
\hline image & GA & DE & PSO & ABC & HS & HMS \\
\hline 12003 & 3.6610 & 3.6636 & 3.6662 & 3.6643 & 3.6648 & $\mathbf{3 . 6 5 9 3}$ \\
42049 & 2.7690 & 2.7628 & 2.7478 & 2.8080 & 2.7547 & $\mathbf{2 . 7 3 9 5}$ \\
181079 & 3.2142 & 3.1147 & 3.2087 & 3.1713 & 3.1619 & $\mathbf{2 . 9 0 0 3}$ \\
198054 & 2.3013 & 2.2529 & 2.3399 & 2.3463 & 2.2985 & $\mathbf{1 . 9 7 2 6}$ \\
385028 & 3.8303 & 3.8159 & 3.8045 & 3.8070 & 3.8160 & $\mathbf{3 . 5 3 2 6}$ \\
\hline average rank & 4.60 & 3.00 & 4.00 & 4.60 & 3.80 & 1.00 \\
\hline
\end{tabular}

The probabilistic Rand index (PRI) [42] calculates the ratio of pairs of pixels whose labellings are consistent between the computed and the ground truth segmentations, with a higher PRI indicating better performance. Table 18 gives the PRI results for all algorithms and shows that for all images our HMS method yields the best segmentation, clearly outperforming the other algorithms.

Table 18: PRI results for Berkeley images and all algorithms. Best results are bolded.

\begin{tabular}{lcccccc}
\hline image & GA & DE & PSO & ABC & HS & HMS \\
\hline 12003 & 0.6329 & 0.6306 & 0.6320 & 0.6355 & 0.6360 & $\mathbf{0 . 6 3 7 1}$ \\
42049 & 0.6670 & 0.6664 & 0.6594 & 0.6692 & 0.6610 & $\mathbf{0 . 6 6 9 6}$ \\
181079 & 0.5968 & 0.5989 & 0.5982 & 0.5925 & 0.5973 & $\mathbf{0 . 6 0 1 3}$ \\
198054 & 0.7206 & 0.7207 & 0.7234 & 0.7262 & 0.7264 & $\mathbf{0 . 7 3 7 4}$ \\
385028 & 0.6028 & 0.6015 & 0.6024 & 0.6052 & 0.6066 & $\mathbf{0 . 6 1 6 1}$ \\
\hline average rank & 4.4 & 4.6 & 4.6 & 3.4 & 3 & 1 \\
\hline
\end{tabular}




\section{Conclusions}

In this paper, we have proposed a novel algorithm for image clustering based on the human mental search (HMS) algorithm. Our HMS approach to image clustering encodes the cluster centres as candidate solutions, and we investigate three objective functions to asses the quality of solutions.

To demonstrate the potential and effectiveness of the proposed algorithm, an extensive set of experiments are provided based on a variety of measures that include objective function criteria, a clustering validity index, non-parametric statistical analysis, stability analysis, and - importantly - several image segmentation metrics. In addition, the proposed algorithm is compared with five other metaheuristics algorithms (a genetic algorithm, differential evolution, particle swarm optimisation, artificial bee colony algorithm, and harmony search) as well as to three conventional clustering-based methods ( $k$-means, fuzzy $c$-means, and Gaussian mixture model). The experimental results demonstrate that our approach clearly, and statistically, outperforms other algorithms confirming our HMS image clustering algorithm to yield a powerful tool for image segmentation. We have also shown our approach to be more robust and to provide faster convergence compared to other methods, as well being able to achieve further improvements through optimising the parameters of the algorithm.

The proposed algorithm applies $k$-means to cluster the population in each iteration. $k$-means is a time-consuming task, especially as it is applied in each iteration. Investigating mechanisms with lower computational burden will be considered in futuristic research. While in this paper we only investigated segmentation of greyscale images, it is clearly straightforward to extend our algorithm to colour images, while other planned future work includes the combination of our approach with local search operators to further improve its performance, the application of alternative objective functions to improve segmentation, and to incorporate a mechanism for automatic determination of the number of clusters (image regions).

\section{References}

[1] S. Suresh and S. Lal, An efficient cuckoo search algorithm based multilevel thresholding for segmentation of satellite images using different objective functions, Expert Systems with Applications, 58, 184-209, 2016.

[2] A.K. Bhandari, A. Kumar, G.K. Singh, Modified artificial bee colony based computationally efficient multilevel thresholding for satellite image segmentation using Kapur's, Otsu and Tsallis functions, Expert Systems with Applications, 42, 1573-1601, 2015

[3] G. Schaefer, Soft computing-based colour quantisation, EURASIP Journal on Image and Video Processing, 8, 2014.

[4] B. Gaonkar, L. Macyszyn, M. Bilello, M.S. Sadaghiani, H. Akbari, M.A. Attiah, Z.S. Ali, X. Da, Y. Zhan, D. O'Rourke, Automated tumor volumetry using computer-aided image segmentation, Academic Radiology, 22, 653$661,2015$.

[5] S. Bargoti, J.P. Underwood, Image segmentation for fruit detection and yield estimation in apple orchards, Journal of Field Robotics, 2017. 
[6] S.H.R. Sanei, R.S. Fertig, Uncorrelated volume element for stochastic modeling of microstructures based on local fiber volume fraction variation, Composites Science and Technology, 117, 191-198, 2015

[7] A.K. Jain, Data clustering: 50 years beyond k-means, Pattern Recognition Letters, 31, 651-666, 2010.

[8] D. Karaboga, C. Ozturk, A novel clustering approach: Artificial Bee Colony (ABC) algorithm, Applied Soft Computing, 11, 652-657, 2011.

[9] D. Whitley, A genetic algorithm tutorial, Statistics and Computing, 4, 65-85, 1994.

[10] R. Eberchart, J. Kennedy, Particle swarm optimization, IEEE International Conference on Neural Networks, 1995.

[11] R. Storn, K. Price, Differential evolution-a simple and efficient heuristic for global optimization over continuous spaces, Journal of Global Optimization, 11, 341-359, 1997.

[12] D. Karaboga, B. Basturk, A powerful and efficient algorithm for numerical function optimization: artificial bee colony (ABC) algorithm, Journal of Global Optimization, 39, 459-471, 2007

[13] Z.W. Geem, J.H. Kim, G. Loganathan, A new heuristic optimization algorithm: harmony search, Simulation, 76 (2001) 60-68.

[14] C. Ozturk, E. Hancer, D. Karaboga, Improved clustering criterion for image clustering with artificial bee colony algorithm, Pattern Analysis and Applications, 18, 587-599, 2015.

[15] S.J. Mousavirad, H. Ebrahimpour-Komleh, Multilevel image thresholding using entropy of histogram and recently developed population-based metaheuristic algorithms, Evolutionary Intelligence, 10, 45-75, 2017.

[16] S. Pare, A.K. Bhandari, A. Kumar, G.K. Singh, A new technique for multilevel color image thresholding based on modified fuzzy entropy and Lévy flight firefly algorithm, Computers \& Electrical Engineering, 70, 476-495, 2018.

[17] M. Awad, K. Chehdi, A. Nasri, Multi-component image segmentation using a hybrid dynamic genetic algorithm and fuzzy c-means, IET Image Processing, 3, 52-62, 2009.

[18] A. Khan, M.A. Jaffar, Genetic algorithm and self organizing map based fuzzy hybrid intelligent method for color image segmentation, Applied Soft Computing, 32, 300-310, 2015.

[19] Y. Shi, R. Eberhart, A modified particle swarm optimizer, IEEE Congress on Evolutionary Computation, pp. 69-73, 1998.

[20] M. Omran, A. Salman, A.P. Engelbrecht, Image classification using particle swarm optimization, 4th Asia-Pacific Conference on Simulated Evolution and Learning, pp. 18-22, 2002.

[21] J. Yu, A Novel Chaos PSO Clustering Algorithm for Texture Image Segmentation, Recent Advances in Computer Science and Information Engineering, 269-274, 2012.

[22] M. Omran, S. Al-Sharhan, Barebones particle swarm methods for unsupervised image classification, IEEE Congress on Evolutionary Computation, pp. 3247-3252, 2007. 
[23] W. Jian-Xiang, S. Yue-Hong, T. Zhao-Ling, Image clustering segmentation based on fuzzy mutual information and PSO, Applied Informatics and Communication, (2011) 1-12.

[24] M. Omran, A.P. Engelbrecht, A. Salman, Particle swarm optimization method for image clustering, International Journal of Pattern Recognition and Artificial Intelligence, 19, 297-321, 2005.

[25] M. Omran, A. Engelbrecht, A. Salman, Particle swarm optimization for pattern recognition and image processing, Swarm Intelligence in Data Mining, 125-151, 2006.

[26] S. Mukhopadhyay, P. Mandal, T. Pal, J.K. Mandal, Image clustering based on different length particle swarm optimization (DPSO), 3rd International Conference on Frontiers of Intelligent Computing: Theory and Applications pp. 711-718, 2014.

[27] S. Das, A. Abraham, A. Konar, Automatic hard clustering using improved differential evolution algorithm, Metaheuristic Clustering, 137-174, 2009.

[28] S. Das, A. Konar, Automatic image pixel clustering with an improved differential evolution, Applied Soft Computing, 9, 226-236, 2009.

[29] W. Kwedlo, A clustering method combining differential evolution with the k-means algorithm, Pattern Recognition Letters, 32, 1613-1621, 2011.

[30] C. Ozturk, E. Hancer, D. Karaboga, Dynamic clustering with improved binary artificial bee colony algorithm, Applied Soft Computing, 28, 69-80, 2015.

[31] L. Wang, Y. Yufeng, J. Liu, Clustering with a novel global harmony search algorithm for image segmentation, International Journal of Hybrid Information Technology, 9, 183-194, 2016.

[32] S.J. Mousavirad, H. Ebrahimpour-Komleh, Human mental search: a new population-based metaheuristic optimization algorithm, Applied Intelligence, 1-38, 2017.

[33] D. H. Wolpert and W. G. Macready, ”No free lunch theorems for optimization,” IEEE transactions on evolutionary computation, 1, 67-82, 1997.

[34] P. Arbelaez, C. Fowlkes, D. Martin, The Berkeley segmentation dataset and benchmark, http://www.eecs. berkeley.edu/Research/Projects/CS/vision/bsds

[35] D. Karaboga, B. Akay, A comparative study of artificial bee colony algorithm, Applied Mathematics and Computation, 214, 108-132, 2009.

[36] P.N. Suganthan, N. Hansen, J.J. Liang, K. Deb, Y.-P. Chen, A. Auger, S. Tiwari, Problem definitions and evaluation criteria for the CEC 2005 special session on real-parameter optimization, KanGAL report 2005005, 2005.

[37] D.L. Davies, D.W. Bouldin, A cluster separation measure, IEEE Transactions on Pattern Analysis and Machine Intelligence, 224-227, 1979. 
[38] S.-H. Liu, M. Mernik, D. HrnčIč, M. Črepinšek, A parameter control method of evolutionary algorithms using exploration and exploitation measures with a practical application for fitting Sovova's mass transfer model, Applied Soft Computing, 13, 3792-3805, 2013.

[39] M. Borsotti, P. Campadelli, R. Schettini, Quantitative evaluation of color image segmentation results, Pattern Recognition Letters, 19, 741-747, 1998.

[40] M.D. Levine, A.M. Nazif, Dynamic measurement of computer generated image segmentations, IEEE Transactions on Pattern Analysis and Machine Intelligence, 155-164, 1985.

[41] M. Meilă, Comparing clusterings: an axiomatic view, 22nd International Conference on Machine Learning, pp. $577-584,2005$.

[42] C. Pantofaru, M. Hebert, A comparison of image segmentation algorithms, Robotics Institute, 336, 2005. 